TABLE 1DR. Compilation of geochronologic ages of Laramide rocks of Sonora east of isochron 70 Ma shown in Figure 14.

\title{
VOLCANIC ROCKS
}

\begin{tabular}{|c|c|c|c|}
\hline ROCK & AGE & METHOD & COORDINATES \\
\hline UAKA 78-20 (rhyo) & $40.6 \pm 1.1$ & $\mathrm{~K}-\operatorname{Ar}(\mathrm{wr})$ & $29^{\circ} 43^{\prime} 18^{\prime \prime N}$; 1094'15"W \\
\hline Andesite & $43.2 \pm 1.1$ & $\mathrm{~K}-\mathrm{Ar}(\mathrm{wr})$ & $30^{\circ} 24^{\prime} ; 109^{\circ} 42^{\prime}$ \\
\hline Dacite & $43.7 \pm 0.2$ & ${ }^{40} \mathrm{Ar} /{ }^{39} \mathrm{Ar}(\mathrm{pl})$ & \\
\hline Latite & $52.5 \pm 1.3$ & K-Ar (bi) & \\
\hline Volcanic rock & $52.9 \pm 0.9$ & $\mathrm{~K}-\mathrm{Ar}(\mathrm{wr})$ & \\
\hline Riolite & $54.3 \pm 0.92$ & ${ }^{40} \mathrm{Ar} /{ }^{39} \mathrm{Ar}(\mathrm{pl})$ & \\
\hline Riolite & $55.3 \pm 2.1$ & K-Ar (bi) & $28^{\circ} 27^{\prime} 28^{\prime \prime} \mathrm{N} ; 109^{\circ} 21^{\prime} 32^{\prime \prime} \mathrm{E}$ \\
\hline Riolite & $56.8 \pm 0.9$ & $\mathrm{~K} / \mathrm{Ar}(\mathrm{bi})$ & $28^{\circ} 24^{\prime} 15^{\prime \prime} \mathrm{N} ; 109^{\circ} 14^{\prime} 13^{\prime \prime}$ \\
\hline Andesite & $56.7 \pm 1.2$ & ${ }^{40} \mathrm{Ar} /{ }^{39} \mathrm{Ar}(\mathrm{bi})$ & 573087E; 3413081N \\
\hline Dacite & $58.67 \pm 0.17$ & ${ }^{40} \mathrm{Ar} /{ }^{39} \mathrm{Ar}(\mathrm{bi})$ & \\
\hline Andesite & $60 \pm 0.5$ & ${ }^{40} \mathrm{Ar} /{ }^{39} \mathrm{Ar}(\mathrm{pl})$ & \\
\hline Andesite & $61.2 \pm 3.4$ & $\mathrm{~K} / \mathrm{Ar}(\mathrm{pl})$ & $28^{\circ} 24^{\prime} 45^{\prime \prime} \mathrm{N} ; 109^{\circ} 05^{\prime} 10^{\prime \prime} \mathrm{E}$ \\
\hline Andesite & $64.2 \pm 2.5$ & $\mathrm{U}-\mathrm{Pb}(\mathrm{zr})$ & \\
\hline Andesite & $65.7 \pm 2.5$ & K-Ar (bi) & \\
\hline Volcanic rock & $65.9 \pm 0.4$ & $\mathrm{~K}-\mathrm{Ar}(\mathrm{wr})$ & \\
\hline Riodacite & $65.8 \pm 0.4$ & ${ }^{40} \mathrm{Ar} /{ }^{39} \mathrm{Ar}(\mathrm{bi})$ & 576331E; 3421872N \\
\hline Riodacite & $66.0 \pm 0.4$ & ${ }^{40} \mathrm{Ar} /{ }^{39} \mathrm{Ar}(\mathrm{bi})$ & $30^{\circ} 55^{\prime} 44^{\prime \prime} ; 110^{\circ} 12^{\prime} 03^{\prime \prime}$ \\
\hline Dacite & $69.1 \pm 0.40$ & ${ }^{40} \mathrm{Ar} /{ }^{39} \mathrm{Ar}(\mathrm{bi})$ & $30^{\circ} 53^{\prime} 13^{\prime \prime} ; 110^{\circ} 13^{\prime} 00^{\prime \prime}$ \\
\hline Rhyodacite & $69 \pm 0.2$ & ${ }^{40} \mathrm{Ar} /{ }^{39} \mathrm{Ar}(\mathrm{bi})$ & \\
\hline Dacite & $69.1 \pm 0.4$ & ${ }^{40} \mathrm{Ar} /{ }^{39} \mathrm{Ar}(\mathrm{bi})$ & 574863E; 3417160N \\
\hline Ignimbrite & $69.7 \pm 0.6$ & $\mathrm{U}-\mathrm{Pb}(\mathrm{zr})$ & \\
\hline Crystal tuff & $70.2 \pm 0.6$ & $\mathrm{U}-\mathrm{Pb}(\mathrm{zr})$ & \\
\hline Tuff & $71 \pm 0.35$ & ${ }^{40} \mathrm{Ar} /{ }^{39} \mathrm{Ar}(\mathrm{san})$ & $31^{\circ} 10^{\prime} 17^{\prime \prime}, 110^{\circ} 15^{\prime} 47^{\prime \prime}$ \\
\hline Andesite & $72.6 \pm 1.2$ & ${ }^{40} \mathrm{Ar} /{ }^{39} \mathrm{Ar}(\mathrm{bi})$ & $30^{\circ} 54^{\prime} 28^{\prime \prime}$ and $110^{\circ} 14^{\prime} 24^{\prime \prime}$ \\
\hline Tuff & $72.5 \pm 0.5$ & $\mathrm{U}-\mathrm{Pb}(\mathrm{zr})$ & \\
\hline Tuff & $72.6 \pm 0.8$ & $\mathrm{U}-\mathrm{Pb}(\mathrm{zr})$ & \\
\hline Dacite & $72.6 \pm 1.2$ & ${ }^{40} \mathrm{Ar} /{ }^{39} \mathrm{Ar}(\mathrm{bi})$ & 572635E; 3419487N \\
\hline Andesite & $72.1 \pm 0.4$ & ${ }^{40} \mathrm{Ar} /{ }^{39} \mathrm{Ar}(\mathrm{bi})$ & \\
\hline Tuff & $73.4 \pm 0.18$ & ${ }^{40} \mathrm{Ar} /{ }^{39} \mathrm{Ar}(\mathrm{san})$ & $31^{\circ} 14^{\prime} 57^{\prime \prime} ; 110^{\circ} 22^{\prime} 18^{\prime \prime}$ \\
\hline Andesite & $73.6 \pm 1$ & $\mathrm{U}-\mathrm{Pb}(\mathrm{zr})$ & \\
\hline Volcanic rx & $74.6 \pm 3.7$ & K-Ar (wr) & \\
\hline ? & 76 & $\mathrm{U}-\mathrm{Pb}(\mathrm{zr})$ & \\
\hline Dacite & $89.0 \pm 0.8$ & $\mathrm{U}-\mathrm{Pb}(\mathrm{zr})$ & \\
\hline Tuff? & $90.1 \pm 0.7$ & $\mathrm{U}-\mathrm{Pb}(\mathrm{zr})$ & \\
\hline
\end{tabular}

$\begin{array}{ll}\text { LOCALITY } & \text { AUTHOR } \\ \text { Oposura mine } & \text { Damon et al. (1983) } \\ \text { La Florida } & \text { Damon et al. (1983) } \\ \text { Santa Rosa } & \text { Gans (1997) } \\ \text { La Caridad mine } & \text { Damon et al. (1983) } \\ & \text { Pubellier et al. (1995) } \\ \text { Santa Rosa } & \text { Gans (1997) }\end{array}$

Santa Rosa

Roldan Quintana et al. (2009)

Roldan Quintana et al. (2009)

Damon et al. (1983)

Arizpe , Cerro Las Jarill González-León et al. (2000)

Santa Rosa Gans (1997)

La Caridad mine
Cerro Las Conchas
Cerro Las Conchas

Roldan Quintana et al. (2009)

Valencia et al. (2005)

Pubellier et al. (1995)

Cerro Las Conchas Pubellier and Rangin (1987)

Cox et al. (2006)

USGS (this work)

USGS (this work)

North of Cananea Wodzicki (1995)

Cox et al. (2006)

Central Sonora McDowell et al. (2001)

Central Sonora McDowell et al. (2001)

USGS (this work)

Cananea USGS (this work)

central Sonora McDowell et al. (2001)

central Sonora McDowell et al. (2001)

Cox et al. (2006)

Bacoachi USGS (this work)

USGS (this work)

La Caridad Rascón Heimpel et al., 2010

Cerro Las Conchas Pubellier and Rangin (1987)

"...near El Tuli..." McDowell et al. (2001)

Central Sonora McDowell et al. (2001)

Central Sonora McDowell et al. (2001) 


\section{PLUTONIC ROCKS}

99-98 (g)

Gabbro

UAKA74-162 (po)

P-3 (po)

SO-15 (gd)

$\mathrm{P}-1(\mathrm{mz})$

SO-8 (g)

G-1 (gd)

G-2 (gd)

(qm)

(di)

MV-5

SR-83 (gd)

UAKA73-148 (qm)

CH98-11d (gd)

UAKA 81-02 (di)

SO-63 (gd)

-1 (di)

gd

SO-35 (gd)

SO-5 (gd)

59-96

SO-26 (gd)

(po)

MV-15 (gd)

UAKA81-01 (mz)

UAKA 80-07 (gd)

MV-19

SO-80 (gd)

MA-1 (gd)

MV-10 (gd)

(mz)
$41.8 \pm 0.7$

$\mathrm{U}-\mathrm{Pb}(\mathrm{zr})$

$\mathrm{Rb} / \mathrm{Sr}(\mathrm{hb})$

$3244858 \mathrm{~N} ; 576850 \mathrm{E}$

$3258270 \mathrm{~N} ; 573580 \mathrm{E}$

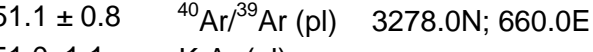

K-Ar (pl)

$51 \pm 6 \quad{ }^{40} \mathrm{Ar} /{ }^{39} \mathrm{Ar}(\mathrm{bi})$

$52.6 \pm 1.6 \quad$ U-Pb (zr)

$54.3 \pm 2.9 \quad \mathrm{~K}-\mathrm{Ar}(\mathrm{hb})$

$54.3 \pm 1.7 \quad \mathrm{U}-\mathrm{Pb}(\mathrm{zr})$

$55.5 \pm 1.9$

$55.6 \pm 1.7$

$55.6 \pm 0.3$

$56.0+5.1$

$56.3 \pm 0.5$

$56.7 \pm 0.2$

$56.8+12$

56.9

56.9

$57 \pm 3$

$57.4 \pm 1.4$

$57.6 \pm 0.9$

$58.3 \pm 2$

$58.2 \pm 3.3$

59

$59.9 \pm 2$

K-Ar (wr)

K-A

$\mathrm{U}-\mathrm{Pb}(\mathrm{zr})$

$\mathrm{U}-\mathrm{Pb}(\mathrm{zr})$

$\mathrm{K}-\operatorname{Ar}(\mathrm{wr})$

$\mathrm{K}-\mathrm{Ar}$ (bi)

${ }^{40} \mathrm{Ar} /{ }^{39} \mathrm{Ar}(\mathrm{hb})$

${ }^{40} \operatorname{Ar} /{ }^{39} \operatorname{Ar}(\mathrm{hb})$

$\mathrm{K}-\mathrm{Ar}$ (bi)

$\mathrm{K}-\operatorname{Ar}(\mathrm{wr})$

$\mathrm{K}-\mathrm{Ar}(\mathrm{wr})$

U-Pb (zr)

$\mathrm{K}-\operatorname{Ar}(\mathrm{mx})$

$\mathrm{K}-\mathrm{Ar}(\mathrm{ms})$

$\mathrm{U}-\mathrm{Pb}(\mathrm{zr})$

$\mathrm{U}-\mathrm{Pb}(\mathrm{zr})$

$\mathrm{K}-\mathrm{Ar}(\mathrm{hb})$

K-Ar (wr)

K-Ar (bi)

$\mathrm{K}-\mathrm{Ar}(\mathrm{wr})$

(h)

$60.5 \pm 0.33$

${ }^{40} \mathrm{Ar} /{ }^{39} \mathrm{Ar}(\mathrm{hb})$

$61.2 \pm 1.4$

$\mathrm{K}-\mathrm{Ar}(\mathrm{wr})$

K-Ar (hb)

$62.0 \pm 1.7$

$62.0 \pm 1$

U-Pb (zr)

$62.0 \pm 3 \quad \mathrm{~K}-\mathrm{Ar}(\mathrm{hb})$

$62.7 \pm 1 \quad \mathrm{~K}-\mathrm{Ar}$ bt)

$62.9 \pm 1.5 \quad \mathrm{~K}-\mathrm{Ar}(\mathrm{hb})$

$63.1 \pm 1.7 \quad$ K-Ar (bi)
$3136.1 \mathrm{~N} ; 643.6 \mathrm{E}$

$3179.5 N ; 557.8 \mathrm{E}$

$3348.3 \mathrm{~N} ; 625.5 \mathrm{E}$

$8^{\circ} 36^{\prime} 03^{\prime \prime} \mathrm{N}$; $109^{\circ} 36^{\prime} 04^{\prime \prime} \mathrm{W}$

$28^{\circ} 24^{\prime} 13^{\prime \prime} \mathrm{N} ; 110^{\circ} 14^{\prime} 30 " \mathrm{~W}$

$3159.9 N ; 631.8 \mathrm{E}$

3173.0N; 513.3E

$28^{\circ} 53^{\prime} 06^{\prime \prime} \mathrm{N}$; $109^{\circ} 48^{\prime} 54^{\prime \prime} \mathrm{W}$

$28^{\circ} 37^{\prime} \mathrm{N} \cdot 109^{\circ} 53^{\prime} 18^{\prime \prime} \mathrm{W}$

$28^{\circ} 53^{\prime} 51$ "N; 10954'16"W

$28^{\circ} 17^{\prime} 10^{\prime \prime} \mathrm{N} ; 109^{\circ} 47^{\prime} 00^{\prime \prime} \mathrm{W}$

$12 \mathrm{R} 3142.4 \mathrm{~N}, 730.2 \mathrm{E}$
Granito Oquimonis González-Becuar (2010)

Puerta del Sol, Ures González-Becuar (2010)

Roldán-Quintana et al., 2009

Las Rastritas ranch Almirudis-Echeverría, 2010

San Felipe

Damon et al., 1983b

Cumobabi mine

La Caridad mine

Zuñiga Hernández, 2010

Valencia et al., 2005

Roldán-Quintana et al., 2009

La Caridad mine

Valencia et al., 2005

Housh and McDowell, 2005

Leon and Miller, 1981

Valencia et al, 2005

La Caridad mine

a Caridad mine

Cumobabi mine

Cumobabi mine

Suaqui

Santa Rosa

Batamote

Bella Esperanza

San Javier

La Caridad mine

Sierra Mazatán

alencia et al., 2005

Scherkenbach et al., 1985

Scherkenbach et al., 1985

Valencia-Moreno et al., 2006

Gans, 1997

Damon et al., 1983

Housh and McDowell, 2005

Housh and McDowell, 2005

Anderson et al., 1980

Damon et al., 1983

Roldán-Quintana et al., 2009

Valencia et al., 2005

Anderson et al. 1980

Roldán-Quintana et al., 2009

Housh and McDowell, 2005

Cananea mine Damon et al 1983a

Maycoba Valencia-Moreno et al., 2006

Housh and McDowell, 2005

Rascón Heimpel et al., 2010

La Caridad

City Hermosillo

Rebeico

Valencia-Moreno et al., 200

Damon et al., 1983

Damon et al 1983

. 1991

Roldán-Quintana et al., 2009

Roldán-Quintana et al., 2009

Damon et al., 1983

Cumobabi mine

Scherkenbach et al., 1985 
Housh and McDowell, 2005 Roldán-Quintana et al., 2009

$\mathrm{CR} \mathrm{H} 12(\mathrm{~g}) \quad 63.6 \pm 3.2 \quad \mathrm{~K} / \mathrm{Ar}(\mathrm{w})$

$\mathrm{M}-120(\mathrm{mz})$

gd

$63.9 \pm 2$

$\mathrm{U}-\mathrm{Pb}(\mathrm{zr})$

UAKA 81-06 (gd)

$64 \pm 3$

$\mathrm{U}-\mathrm{Pb}(\mathrm{zr})$

$\mathrm{SO}-74$ (di)

$64.1+1.4$

K-Ar (bt)

SO-2 (gd)

$64.9 \pm 1.7 \quad \mathrm{~K}-\mathrm{Ar}(\mathrm{bt})$

UAKA $80-20$ (gd)

65

$\mathrm{K}-\mathrm{Ar}(\mathrm{bt})$

SO-7

$\mathrm{K}-\mathrm{Ar}(\mathrm{bt})$

99-SF-04 (g)

$65.9 \pm 1.6 \quad \mathrm{~K}-\mathrm{Ar}(\mathrm{b})$

99-SF-05 (gd)

66.0

$\mathrm{K}-\mathrm{Ar}(\mathrm{wr})$
$\mathrm{U}-\mathrm{Pb}(\mathrm{zr})$

UAKA80-20

$66.5 \pm 0.5$

U-Pb (zr)

$\mathrm{SO}-3$ (gd)

$66.7 \pm 1.6$

$\mathrm{K}-\mathrm{Ar}(\mathrm{bt})$

$3306.6 \mathrm{~N}: 652.0 \mathrm{E}$

Sierra Chiltepin

La Caridad mine

Cuitaca grd

2905'20" N; $110^{\circ} 56^{\prime} 08^{\prime \prime} \mathrm{W}$ Hermosillo

$28^{\circ} 18^{\prime} 15^{\prime \prime} \mathrm{N} ; 110^{\circ} 08^{\prime} 00^{\prime \prime W}$

Hermosillo

$31566.0 \mathrm{~N} ; 606.9 \mathrm{E}$

28050'322N; $110^{\circ} 12^{\prime} 20^{\prime \prime W} \quad$ Cobachi

31.66.3N; 588.0E

487250E; 3370400N

$487150 \mathrm{E} ; 3370400 \mathrm{~N}$

Cobachi

Housh and McDowell, 2005

Pubellier et al., 1995

Valencia Gómez, 2005

Anderson and Silver, 1977

Damon et al., 1983

Roldán-Quintana et al., 2009

Housh and McDowell, 2005

Damon et al., 1983

Housh and McDowell, 2005

Poulsen et al., 2008

Poulsen et al., 2008

Damon et al., 1983

Housh and McDowell, 2005

González-León et al., 2000

$67.97 \pm 0.19{ }^{40} \mathrm{Ar} /{ }^{39} \mathrm{Ar}(\mathrm{bi})$

$68.2 \pm 1.3 \quad{ }^{40} \mathrm{Ar} /{ }^{39} \mathrm{Ar}(\mathrm{bi})$

$3164.7 \mathrm{~N} ; 624.1 \mathrm{E}$

PED-3960 (qm)

$M V-11$ (gd)

$68.7 \pm 1.7$

$3278.0 \mathrm{~N} ; 660.0 \mathrm{E}$

Cobachi

Bacanuchi

Las Rastritas ranch

Torreón

Hermita

99-SF-03 (qm)

68.7 $\pm 1.1 \quad{ }^{40} \mathrm{Ar} /{ }^{39} \mathrm{Ar}(\mathrm{hb})$

$\mathrm{mz}$

$68.3 \pm 3.2$

$\mathrm{U}-\mathrm{Pb}(\mathrm{zr})$

487175E; 3356200N

El Llano mine

Benjamin Hill

Chivato, Cananea

$69 \pm 1 \quad U-P b(z r)$

03-11 (mz) $\quad 88.7 \pm 1 \quad$ U-Pb (zr)

Bacanora

$90.6 \pm 1 \quad$ U-Pb (zr)

Bacanora

Amirudis-Echeverría, 2010

Damon et al., 1983

Valencia-Moreno et al., 2006

Poulsen et al., 2008

Castillón González et al., 2010

Anderson and Silver, 1977

Pérez-Segura et al., 2009

Pérez-Segura et al., 2009

Tn, tonalite; mz, monzonite; qm, quartz monzonite; gd, granodiorite; g, granite; di, diorite; po, porphyry; bi, biotite, hb, hornblende; wr, whole rock; zr, zircon;

pl, plagioclase; mx, matrix; ms, muscovite

\section{REFERENCES}

Almirudis Echeverría, E., 2010, Petrogénesis y geocronología ${ }^{40} \mathrm{Ar} /{ }^{39} \mathrm{Ar}$ del plutonismo Laramídico en el área Sobai Satechi, Sonora [Bachellor thesis]:

Hermosillo, Universidad de Sonora, $103 \mathrm{p}$.

Anderson, T.H., Silver, L.T., 1977, U-Pb isotope ages of granitic plutons near Cananea, Sonora: Economic Geology, v. 72, p. 827-836

Castillón González, J., Herrera Urbina, S., Iriondo, A., and Paz Moreno, F.A., 2010, Petrogénesis de intrusivos laramídicos y Proterozoicos al sur de Benjamin Hill,

Sonora central, México in Valencia-Moreno, M., and Manea, M., eds. La Orogenia Laramide del SW de Norte América, Simposio GeoLaramide, Universidad

Nacional Autónoma de México y Universidad de Sonora, resúmenes, p. 41.

Cox, D.P., Miller, R.J., and Woodburne, K., 2006, The Laramide Mesa Formation and the Ojo de Agua Caldera, Southeast of Cananea Copper Mining District,

Sonora, Mexico: USGS Scientific Investigations Report 2006-5022, 7 p.

Damon, P.E., Shafiqullah, M., Roldán-Quintana, J., and Cochemé, J.J., 1983, El batolito Laramide (90-40 Ma) de Sonora: Asociación de Ingenieros de Minas,

Metalugistas y Geólogos de México (AIMMGM), Memoria técnica XV, p. 63-95.

Gans, P.B., 1997, Large-magnitude Oligo-Miocene extension in southern Sonora: implications for the tectonic evolution of northwest Mexico: Tectonics, 
v. 16 , no. 3, p. 388-408.

González Becuar, E., 2011, Geología, geoquímica y geocronología del área de Puerta del Sol, Sonora central, México [Bachellor thesis]: Hermosillo,

Universidad de Sonora, $65 \mathrm{p}$.

González-León, C., McIntosh, W.C., Lozano-Santacruz, R., Valencia-Moreno, M., Amaya-Martínez, R., and Rodríguez Castañeda, J.L., 2000, Cretaceous and Tertiary sedimentary, magmatic, and tectonics evolution of north-central Sonora (Arizpe and Bacanuchi quadrangles), northwest Mexico: Geological Society of America Bulletin, v. 112, no. 4, p. 600-611.

Housh, T.B., and McDowell, F.W., 2005, Isotope provinces in Laramide and mid-Tertiary igneous rocks of northwestern Mexico (Chihuahua and Sonora) and their relation to basement configuration in Anderson, T. H., Nourse, J. A., McKee, J., and Steiner, M.B., eds., The Mojave-Sonora megashear hypothesis: development, assessment, and alternatives: Geological Society of America Special Paper 393, p. 671-692, doi: 10.1130/2005.2393(25).

León, F., and Miller, J., 1981, Opodepe Molybdenum-Copper Porphyry Deposit, in Ortlieb, L., and Roldán-Quintana, J., eds., Geology of Northwestern Mexico and Southern Arizona: Geological Society of America, Cordilleran Section Annual Meeting, Field Guides and Papers, p. 223-238.

McDowell, F. W., Roldán-Quintana, J., and Connelly, J.N., 2001, Duration of Late Cretaceous-early Tertiary magmatism in east-central Sonora, Mexico: Geological Society of America Bulletin, vol. 113, pp. 521-531, 2001.

Pérez-Segura, E., González-Partida, E., and Valencia, V.A., 2009, Late Cretaceous adakitic magmatism in east-central Sonora, Mexico, and its relation to

Cu-Zn-Ni-Co skarns: Revista Mexicana de Ciencias Geológicas, v. 26, p. 411-427.

Pubellier. M., and Rangin, C., 1987, Mise en evidence d'une phase cenomano-turonien en Sonora central (Mexique). Consequences sur les relations structurales entre domaine cordillerain et domain tethysien: C.R. Acad. Sci. Paris, t. 305, Serie II, p. 1093-1098.

Pubellier, M., Rangin, C., Rascón, B., Chorowicz, J., Bellon, H., 1995, Cenomanian thrust tectonics in the Sahuaripa region, Sonora: implications about

northwestern megashears, in Jacques-Ayala, C., González-León, C.M., Roldán-Quintana, J. (eds.), Studies in the Mesozoic of Sonora and adjacent areas:

Geological Society of America, Special Paper 301, p. 111-120.

Poulsen, K.H., Mortensen, J.K., and Walford, P.C., 2008, San Francisco gold deposit, Santa Ana region, Sonora, Mexico: Laramide orogenic, intrusion-related mineralization? In Spencer, J.E., and Titley, S.R., eds., Ores and orogenesis: Circum-Pacific tectonics, geologic evolution, and ore deposits: Arizona Geological Society Digest, v. 22, p. 547-559

Rascón Heimpel, M., Valencia-Moreno, M., Ochoa Landin, L., Calmus, T., and Martínez Muller, R., 2010, Geología y geoquímica del prospecto pórfido cuprífero Los Alisos, distrito minero de Nacozari, Sonora, México in Valencia-Moreno, M., and Manea, M., eds. La Orogenia Laramide del SW de Norte América, Simposio GeoLaramide, Universidad Nacional Autónoma de México y Universidad de Sonora, resúmenes, p. 37.

Roldán-Quintana, J., McDowell, F. W., Delgado-Granados, H., and Valencia-Moreno M., 2009, East-west variations in age, chemical and isotopic composition of the Laramide batholith in southern Sonora, Mexico: Revista Mexicana de Ciencias Geológicas, v. 26, p. 543-563.

Scherkenbach, D.A., Sawkins, F. J., and Seyfried,W. E., Jr., 1985. Geologic, fluid inclusions, and geochemical studies of the mineralized breccias at Cumobabi, Sonora, Mexico: Economic Geology, vol. 80, p. 1566-1592.

Valencia, V.A., Ruiz, J., Barra, F., Geherls, G.E., Ducea, M., Titley, S.R., and Ochoa-Landin, L., 2005, U-Pb zircon and Re-Os molybdenite geochronology from La Caridad porphyry copper deposit: insights for the duration of magmatism and mineralization in the Nacozari District, Sonora, Mexico: Mineralium Deposita, $\mathrm{V}$.

40, p. 175-191.

Valencia-Moreno, M.A., Iriondo, A., and González-León, C.M., 2006, Temporal constraints on the eastward migration of the Late Cretaceous-Early Tertiary magmatic arc of NW Mexico based on Ar/Ar hornblende geochronology of granitic rocks: Journal of South American Earth Sciences, v. 22, p. 22-38.

Wodzicki, W.A., 1995, The evolution of Laramide igneous rocks and porphyry copper mineralization in the Cananea district, Sonora, Mexico [Ph.D. thesis]

Tucson, The University of Arizona, $181 \mathrm{p}$

Zuñiga Hernández, L.G., 2010, Estudio geológico, geoquímico y metalognético del cuadrángulo Huépac-Moctezuma, centro-norte de Sonora, México [Ms. thesis]: Hermosillo, Universidad de Sonora, $126 \mathrm{p}$ 